\title{
Preventing Hypothermia in Preterm Infants: A Program of Research
}

\author{
Robin B. Knobel-Dail* \\ Duke University School of Nursing and School of Medicine
}

\begin{abstract}
Neonatal hypothermia is a worldwide problem and leads to increased morbidity and mortality in newborn infants. This paper describes a program of research to examine thermoregulation in premature infants and to decrease neonatal hypothermia. Our studies include 1) examining an intervention to reduce heat loss in premature infants by placing them in polyurethane bags after birth; 2) a descriptive study examining central and peripheral temperatures during the first 12 hours of age; 3) and a study examining central and peripheral temperatures over premature infants' first two weeks of life. Currently we are using an esophageal tube with an imbedded thermistor (Philips Healthcare) to display continuous central temperature in a randomized controlled trial to see if this intervention will lead to warmer temperatures during the stabilization period than monitoring temperatures intermittently using axillary temperatures and skin probes. Future research will include expanding collaborative studies into low to moderate resource countries to help reduce infant hypothermia and infant mortality.
\end{abstract}

Keywords: neonatal hypothermia, thermoregulation, infant body temperature regulation

\section{Introduction}

Neonatal hypothermia has long been a problem associated with increased morbidity and mortality in infants. My first encounter with severe hypothermia was in the early 1990s, when I was working as a neonatal nurse practitioner. The infant I was transporting from a small community hospital to our facility had a body temperature of only $32.7^{\circ} \mathrm{C}$, because care providers had forgotten to turn on the radiant warmer while paying close attention to the infant's cardiac and respiratory problems. The infant survived the transport but died 2 weeks later, most likely from complications related to hypothermia. Neonatal hypothermia is a worldwide problem. Prevalence rates vary widely, ranging from 32 to $85 \%$ (Lunze, Bloom, Jamison, \& Hamer, 2013). My program of research is dedicated to the prevention of neonatal hypothermia and the study of thermoregulation in premature infants to improve infant health throughout the world.

\section{The Premature Infant}

Infants born less than 37 weeks gestational age are considered premature; infants born less than 32 weeks gestation have immature thermoregulation which makes them even more vulnerable to heat loss (Power \& Blood, 2011). Premature infants typical- ly rest with arms and legs extended and have large heads proportional to their bodies-these factors increase the surface area exposed to cold environmental temperatures, thereby increasing heat loss. Because premature infants are vulnerable to heat loss, care must be taken to protect infants from heat loss and maximize their ability to retain heat.

\section{Non-shivering Thermogenesis}

Infants produce heat by non-shivering thermogenesis (NST) until 12 months of age and do not have the ability to shiver, as do adults. Once the hypothalamus receives signals that the infant is experiencing cold, impulses are sent triggering chemical thermogenesis. If an infant experiences cold on the central and peripheral skin receptor sites, signals are sent which cause vasoconstriction in the peripheral vessels, sending blood and heat centrally to protect the central body from hypothermia. NST occurs in the mitochondria of the brown fat and uses uncoupling protein or thermogenin to dissipate heat by uncoupling protons in ATP synthesis (Power \& Blood, 2011).

Premature infants have inefficient heat production by NST and have an inability to exhibit peripheral vasoconstriction either due to immaturity or poor vasomotor tone (Knobel, Holditch-Davis, Schwartz, \&

*Corresponding author: robin.knobel@duke.edu 
Wimmer, 2009). Infants less than about 32 weeks are deficient in thermogenin, have decreased brown fat, and have low amounts of the enzyme 5'-monodeiodinase (necessary for the conversion of T4 to T3)all elements necessary for heat production (Power \& Blood, 2011). Therefore, premature infants are more vulnerable to hypothermia, but all infants may experience hypothermia if heat loss is not prevented.

\section{Mechanisms of Heat Loss}

Heat may be lost through radiation, evaporation, conduction, and convection. Understanding how heat is lost through these mechanisms will allow care providers to minimize hypothermia in neonates.

All body surfaces will emit heat in the form of electromagnetic waves, which will cause nearby surfaces to increase or decrease temperature from this emitted heat. Heat will be lost depending on the temperature difference between the infant's body and the nearby surface. For example, heat can be transferred from the infant's body to a colder window near the infant. Alternatively, heat may be absorbed into the infant's skin from a heat source near the infant, such as a heat lamp. After the first week of life, radiation becomes the most important route of heat loss in premature infants and is as high as 60\% of total heat loss in adults (Sedin, 1995).

Heat may also be transferred by way of conduction when the infant's skin touches solid objects of a different temperature. Care providers should ensure that all surfaces that touch the infant are warmed so as not to increase heat loss from the infant. Heat moves from molecule to molecule when molecules from the skin surface and another surface collide. Air, water, or a solid surface may cause heat exchange when contacting an infant.

When air or fluid moves across the infant's body surface, heat is transferred by convection. If the body surface is warmer than the surrounding environmental air or fluid, heat is first conducted into the air or water, and then swept away by convective air currents or the movement of fluid. Warm molecules are less dense and therefore rise into the air from the skin. Cold water rinsing against an infant, such as with a bath that is not heated, can cause molecules of heat to leave an infant's body and wash away with the water. An infant can lose heat to the air when moved through a cold room such as after birth in a cool room or if placed close to an open window.
Heat is lost through evaporation when water is transmitted through the skin or the respiratory tract. Evaporative heat loss is the largest source of heat loss in infants (Hammarlund, Stomberg, \& Sedin, 1986). Transepidermal water loss has also been found to be inversely related to gestational age. Infants that are cared for in dry environments such as incubators without humidity or under radiant warmers may have increased losses from evaporation (Agren, Sjors, \& Sedin, 2006).

\section{Normal body temperature-What do we want?}

The main goal of achieving thermal stability in infants is to maintain a normal body temperature. Normal body temperature in premature infants has not been described well. Temperature will vary depending on the site of measurement and the device used. General temperature ranges for premature infants by site are given in neonatal textbooks: rectal $\left(36.5^{\circ}-37.5^{\circ} \mathrm{C}\right)$, skin $\left(36.2^{\circ}-37.2^{\circ} \mathrm{C}\right)$, and axillary $\left(36.5^{\circ}-37.3^{\circ} \mathrm{C}\right)$ (Rutter, 2005). The latest standards by the American Academy of Pediatrics/American Congress of Obstetricians and Gynecologists (2012) dictate an axillary temperature of $36.5^{\circ} \mathrm{C}$ in the delivery room and a range of $36.5^{\circ}$ to $37.4^{\circ} \mathrm{C}$ prior to discharge for an infant in an open crib in clothing. A minimal safe axillary temperature of $36.5^{\circ} \mathrm{C}$ should be adequate for infants in a NICU.

Neonatal hypothermia limits have been defined by various researchers and organizations over the years. The World Health Organization (WHO) (1997) has given ranges for the normal temperature of an infant as $36.5^{\circ}$ to $37.5^{\circ} \mathrm{C}$; hypothermia occurs when an infant's temperature decreases below $36.5^{\circ} \mathrm{C}$. WHO also defines mild hypothermia as a body temperature between $36.0^{\circ}-36.5^{\circ} \mathrm{C}$; moderate hypothermia as a temperature between $32.0^{\circ}-36.0^{\circ} \mathrm{C}$ and severe hypothermia is as a temperature less than $32.0^{\circ} \mathrm{C}$. Most current studies are using WHO definitions of hypothermia; infants' body temperature should be kept above a minimal standard of $36.5^{\circ} \mathrm{C}$.

Because we are aware of the mechanisms of heat loss, we know what temperature to strive for and what defines hypothermia in an infant. Studies can be designed to minimize heat loss and aim for thermal stability. My first study was a randomized controlled trial that sought to minimize heat loss after birth by preventing heat loss by evaporation, while allowing radiative heat to warm the infant. 


\section{Using plastic barriers to prevent heat loss}

Like many institutions, we had a high incidence of hypothermic body temperatures in premature infants on admission to our NICU in 1999 (Knobel, Wimmer, \& Holbert, 2005). In a chart review, we found that $93 \%$ of 100 extremely low birth weight (ELBW) infants ( $<1000$ grams at birth) had rectal temperatures less than $36.4^{\circ} \mathrm{C}$ on admission to the NICU using the 1988 American Academy of Pediatrics' definition of hypothermia at the time. Our chart audit led to my first study to prevent heat loss and began my program of research around infant thermal stability. In a randomized controlled trial, we placed half the study infants into polyurethane bags, while they were still wet with amniotic fluid, up to their necks just after birth in the delivery room. The remaining infants received standard care according to the neonatal resuscitation program protocols (NRP) in effect in 1999. We found that infants placed in plastic bags were less likely than infants receiving standard care to have a body temperature less than $36.4^{\circ} \mathrm{C}$ on admission, $(44 \%$ vs. $70 \%(\mathrm{p}<.01))$ and the intervention group had a higher mean admission temperature, $36.5^{\circ} \mathrm{C}$ vs. $36.0^{\circ} \mathrm{C}(\mathrm{p}<.003)$ (Knobel et al., 2005). We attempted to control delivery room temperature at $26^{\circ} \mathrm{C}$; however, we were unsuccessful. Our intervention effect remained significant $(\mathrm{p}<$.0001) when delivery room temperature was controlled in analysis. Warmer delivery room temperatures $\left(>26^{\circ} \mathrm{C}\right)$ were associated with higher admission temperatures in both intervention and control infants, but only infants in bags born in warmer delivery rooms had a mean admission temperature $>36.4^{\circ} \mathrm{C}$.

Our study was one of the first to test this intervention, but since that time, this practice has become standard for delivery room care of infants born prior to 28 weeks gestational age. Current standards also dictate that environmental air temperature should be kept between $22^{\circ}-26^{\circ} \mathrm{C}$ (Pediatrics \& Gynecologists, 2012). Plastic bags or wrap around an infant form an insulation barrier to prevent evaporative heat loss; however, radiative heat is allowed to warm the infant through the plastic (polyurethane or polyethylene). Using plastic bags or wrap is a low cost way to prevent neonatal hypothermia. This practice is being used in under resourced countries when environmental temperatures cannot be controlled, even in larger, term infants (Lester, Kimani, \& Cartledge, 2014).

\section{Body temperature and stabilization in the NICU}

After completing the study to warm infants on transit from the delivery room to the NICU, I moved forward to examine body temperature in ELBW infants during their first 12 hours of life. Even though we reduced the incidence of hypothermia on admission to the NICU, we found their temperatures decreased during stabilization.

In my next study, I examined body temperature in ten ELBW infants during their first 12 hours of life, from admission in the NICU (Knobel et al., 2009). Abdominal (central) and foot (peripheral) temperatures were measured every minute. This exploratory study aimed to describe maturation of thermoregulation in ELBW infants and the ability of a newborn ELBW infant to exhibit peripheral vasoconstriction. In this study, none of the ten infants displayed peripheral vasoconstriction consistently in their first 12 hours of life. Peripheral vasoconstriction is defined as foot temperature below abdominal temperature by $2^{\circ} \mathrm{C}$. Only one infant showed peripheral vasoconstriction for $9 \%$ of his measurements. Another interesting and main finding of this study was that seven of the ten infants kept their foot temperature higher than their abdominal temperature for about 8 of their 12 hours on average. Other researchers have seen this phenomenon but it is not widely documented (Lyon, Pikaar, Badger, \& McIntosh, 1997). This unusual temperature pattern with the related perfusion is concerning because the infant's core is not protected during episodes of hypothermia. It is not clear from published studies if this temperature pattern is related to immaturity or instability.

In addition to body temperature, I measured each infant's heart rate every minute to examine the relationship between heart rate and body temperature during the stabilization period. These data helped to define the body temperature that was associated with heart rate measures in each infant's normal range; normal heart rate was defined as the $25^{\text {th }}$ to $75^{\text {th }}$ percentile or inner $50 \%$ of heart rate measures from all heart rate measures observed over each infants' 12 hour study period. Results indicated that these infants had more normal heart rates when their abdominal temperature was in the 36.8-36.9 range (Knobel, Holditch-Davis, \& Schwartz, 2010). This finding provides evidence that ELBW infants' temperatures should be kept slightly higher than the WHO's recommended min- 
imal body temperature for infants, most likely due to their inefficient thermoregulation and capacity to lose heat easily.

\section{Examining temperature in the first 2 weeks}

Funding from the National Institute of Health/ National Institute of Nursing Research (1R15NR012157-01) and the Robert Wood Johnson Foundation Nurse Faculty Scholar's Grant (68041) enabled our team to examine $30 \mathrm{VLBW}$ infants using a case study design to explore central and peripheral temperature over their first two weeks of life as the next step in my program of research. Because my last study found that infants were unable to exhibit peripheral vasoconstriction in their first 12 hours of life and mostly kept their peripheral temperatures higher than their central temperatures, I hypothesized that their thermal control must be immature and inefficient right after birth. Methodology in this study allows us to follow infants' capacity for thermoregulation over their first two weeks.

Instrumentation in this study was added to explore peripheral perfusion and overall body temperature; a small pilot study was done to evaluate the methodology prior to implementing the larger study (Knobel, Levy, Katz, Guenther, \& Holditch-Davis, 2013). After institutional review board approval, infants were enrolled right after birth once placed in an incubator. Abdominal and foot temperature was measured every minute for two weeks, for a total of 20,000 measures on each infant. Perfusion index of one foot was measured every minute using a Masimo Radical 7 Pulse oximeter with perfusion index capability. Infants were video recorded continuously for their first 5 days inside their incubator to observe them for 12 procedures/care events that might alter temperature. These data will be entered into analysis with physiological variables. We also used infrared imaging to take an overall thermal image of the infant's body once a day for the first 5 days (Knobel, Guenther, \& Rice, 2011). Images are displayed on a computer screen and each pixel will yield a temperature. The image will give our team an idea of where temperature differentials exist in these infants. All data for each infant is being analyzed using case study analysis; all physiological data and coded video data are analyzed together using SAS $^{\circledR}$ statistical package (SAS, Cary, NC). Analysis is ongoing and results will be reported in future publications.

\section{Future Research}

My team's program of research is evolving into three areas to address neonatal thermal stability and reduce infant hypothermia. The first area will continue to explore the link between central and peripheral temperature and morbidity. Once results from our last study are reported, we should have enough evidence to link the irregular time periods of altered vasomotor tone (when an infant has warmer peripheral temperatures than central temperatures) with instability and morbidity. A study will be designed to examine altered vasomotor tone in relationship to morbidity such as necrotizing enterocolitis and infection as well as during blood transfusions in a large multi-center trial.

Secondly, our team just began a randomized controlled trial funded by Philips Healthcare to investigate the use of the InnerSense gastric tube/temperature probe in $160 \mathrm{VLBW}$ infants to measure esophageal temperature from birth through stabilization. In the intervention group, an InnerSense tube is placed down each infant's esophagus just after birth in the delivery room. The tube is then attached to a small temperature monitor with a continuous display. Care givers can watch the continuous display for hypothermic temperatures and react accordingly to ensure thermal stability during delivery resuscitation and through stabilization. The InnerSense tube is removed at 24 hours of age for each infant. The control group receives standard of care, or temperatures monitored intermittently with axillary thermometers or skin surface probes during the delivery room resuscitation and stabilization in the NICU. The primary outcome is admission temperature and one hour temperature means between intervention and control groups. We hypothesize that infants in the intervention group will be warmer on admission and at one hour than the control group infants because care providers will have a reliable, continuous central temperature available to facilitate optimal thermal care. If this trial is successful, the intervention may become the standard of care in the future.

Lastly, our team is interested in implementing educational and clinical interventions through collaborative research in low to moderate resource countries, such as Rwanda, to decrease the incidence of infant hypothermia and reduce infant mortality. We have begun talks with providers in Kigali and will begin working towards a collaborative working relationship to better infant outcomes. 


\section{Conclusion}

Infant hypothermia remains prevalent, especially in premature infants, in the United States and throughout the developing world. My program of research aims to reduce hypothermia in infants and decrease associated morbidity and mortality. Our team has successfully reduced heat loss and hypothermia placing premature infants in polyurethane bags after birth and is now studying a new device, the InnerSense esophageal tube to monitor central temperature continuously after birth and through stabilization. Additionally, we have found links between alterations in central and peripheral temperature and morbidity in premature infants in their first two weeks of life. There is much more research to be done to improve thermal management in infants. Our future endeavors will translate our expertise in thermal management of infants to developing and low to middle income countries to improve infant outcomes.

\section{References}

Agren, J., Sjors, G., \& Sedin, G. (2006). Ambient humidity influences the rate of skin barrier maturation in extremely preterm infants. The Journal of Pediatrics, 148 , 613-617.

Hammarlund, K., Stomberg, B., \& Sedin, G. (1986). Heat loss from the skin of preterm and fullterm newborn infants during the first weeks after birth. Biology of the Neonate, 50(1), 1-10.

Knobel, R., Guenther, B. D., \& Rice, H. (2011). Thermoregulation and thermography in neonatal physiology and disease. Biological Research for Nursing, 13, 274-282.

Knobel, R., Holditch-Davis, D., \& Schwartz, T. (2010). Optimal body temperature in extremely low birth weight infants using heart rate and temperature as indicators. JOGNN, 39, 3-14.

Knobel, R., Holditch-Davis, D., Schwartz, T., \& Wimmer, J. E. (2009). Extremely low birth weight preterm infants lack vasomotor response in relationship to cold body temperatures at birth. Journal of Perinatology, 29, 814-821.
Knobel, R., Levy, J., Katz, L., Guenther, B., \& Holditch-Davis, D. (2013). A pilot study to examine maturation of body temperature control in preterm infants. JOGNN, 42(5), 562-574.

Knobel, R. B., Wimmer, J. E., \& Holbert, D. (2005). Heat loss prevention for preterm infants in the delivery room. Journal of Perinatology, 25(5), 304-308.

Lester, M., Kimani, W., \& Cartledge, P. (2014). Question 1: is the use of plastic bags for thermoregulation in term neonates effective in preventing hypothermia in a low-resource setting? Archives of Disease in Childhood, 99(12), 1169-1172.

Lunze, K., Bloom, D. E., Jamison, D. T., \& Hamer, D. H. (2013). The global burden of neonatal hypothermia: systematic review of a major challenge for newborn survival. BioMed Central Medicine, 11(24), 1-11.

Lyon, A. J., Pikaar, M. E., Badger, P., \& McIntosh, N. (1997). Temperature control in very low birthweight infants during first five days of life. Archives in Diseases of Childhood: Fetal/Neonatal Edition, 76(1), F47-50.

Pediatrics, A. A. o., \& Gynecologists, T. A. C. о. O. a. (2012). Guidelines for Perinatal Care (Vol. seventh edition). Elk Grove Village, IL: American Academy of Pediatrics.

Power, G. G., \& Blood, A. B. (2011). Thermoregulation. In R. A. Polin, W. W. Fox \& S. H. Abman (Eds.), Fetal and Neonatal Physiology (4th ed., Vol. 1, pp. 615-648). Philadelphia, PA: Elservier Saunders.

Rutter, N. (2005). Temperature control and its disorders. In J. M. Rennie (Ed.), Robertson's Textbook of Neonatology (4th ed.). London: Churchill Livingstone.

Sedin, G. (1995). Neonatal heat transfer, routes of heat loss and heat gain. In A. Okken \& J. Koch (Eds.), Thermoregulation of Sick and Low Birth Weight Neonates (pp. 2136). Berlin, Germany: Springer-Verlag.

World Health Organization, D. o. R. H. a. R. (1997). Protection of the newborn: a practical guide. Retrieved 06/04/2014 from http://whqlibdoc.who.int/ hq/1997/WHO_RHT_MSM_97.2.pdf 